Expert Opin Drug Metab Toxicol. 2015 March ; 11(3): 461-470. doi:10.1517/17425255.2015.1000860.

\title{
Denosumab for the Treatment of Osteoporosis
}

\author{
Sarah Zaheer ${ }^{1}$, Meryl LeBoff ${ }^{1}$, and E. Michael Lewiecki ${ }^{2}$ \\ ${ }^{1}$ Brigham and Women's Hospital, Boston, MA, USA \\ ${ }^{2}$ New Mexico Clinical Research \& Osteoporosis Center, Albuquerque, NM, USA
}

\begin{abstract}
Introduction-Low trauma fractures due to osteoporosis are a major health concern worldwide. Despite the availability of many therapeutic compounds to reduce fracture risk, osteoporosis remains undertreated and the burden of osteoporotic fractures remains high. Denosumab is a novel agent that acts to reduce bone turnover, improve bone mineral density, and reduce fracture risk, offering a favorable efficacy and safety profile.
\end{abstract}

Areas covered-This review covers the pharmacology and major clinical trials with extension/ post-marketing follow-up, including trials for all FDA-approved indications of denosumab to date.

Expert Opinion-Denosumab is an efficacious and safe osteoporosis treatment option, with current data up to 8 years of continued use showing continued improvement in bone density with sustained fracture risk reduction. Safety profiles overall are similar to placebo, with no new safety concerns in extension trials, though a theoretical increased risk of infection exists with RANKL inhibition. Future considerations include safety of prolonged treatment beyond 8 years, and efficacy/fracture risk after discontinuation or with non-adherence, given the characteristic pharmacodynamic profile of denosumab.

\section{Keywords}

denosumab; $\mathrm{mAb}$; osteoporosis; receptor activator of nuclear factor kappa-B ligand; treatment

\section{Introduction}

In the United States (US), an estimated 54 million individuals are at risk for low trauma fractures associated with osteopenia or osteoporosis, making it a major U.S. public health care concern [1]. Given the projected demographic changes in the USA, it is expected that the current rates of hip and other fractures in men and women $>50$ years of age will increase by $50 \%$ by 2025 [2]. The clinical sequelae of osteoporotic fractures are significant:

\footnotetext{
Corresponding author: E. Michael Lewiecki, MD, New Mexico Clinical Research \& Osteoporosis Center, 300 Oak St. NE, Albuquerque, NM 87106, USA, Phone 505-938-2117, Fax 505-884-4006, mlewiecki@ gmail.com.

Declaration of interest

S Zaheer has no conflict of interest. M LeBoff has stock ownership of Amgen. She serves on the Board of Trustees of the National Osteoporosis Foundation (no monetary remuneration). EM Lewiecki has received institutional grant/research support from Amgen, Merck, and Eli Lilly; he has served on scientific advisory boards for Amgen, Merck, Eli Lilly, Radius Health, AgNovos Healthcare, and Alexion. He serves on the Board of Trustees of the National Osteoporosis Foundation (no monetary remuneration). The authors have no other relevant affiliations or financial involvement with any organization or entity with a financial interest in or financial conflict with the subject matter or materials discussed in the manuscript apart from those disclosed.
} 
osteoporotic hip fractures are associated with functional limitations and an excess mortality risk of 15-25\% [3], and vertebral fractures are associated with a decreased quality of life, as well as an increase in mortality $[4,5]$. Based on the current U.S. National Osteoporosis Foundation guidelines, an estimated $20 \%$ of men and $37 \%$ of women over age 50 years are potential candidates for treatment to prevent fractures [6].

Although numerous medications are approved for treatment and prevention of osteoporosis, many patients at high risk for fracture are not being treated [7]. Furthermore, when treatment is started, adherence is often poor [8]. There have been concerns about long-term (beyond 3-4 years) efficacy and safety with osteoporosis treatments that may contribute to poor adherence [9].

Denosumab (Box 1) is a novel biologic agent for the treatment of osteoporosis. Up to 8 years of safety and efficacy data are now available from the extension of the pivotal FREEDOM trial, providing longer-term bone density, fracture reduction, and safety/ tolerability data to help guide clinicians in its optimal use in the management of patients at risk for fracture.

\section{Overview of the market}

Current FDA-approved pharmacological agents for use in osteoporosis and fracture risk reduction include bisphosphonates (alendronate, ibandronate, risedronate, and zoledronic acid) [10-13], salmon calcitonin [14], estrogen therapy with or without medroxyprogesterone [15, 16], raloxifene [17], conjugated estrogen/bazedoxifene [18], recombinant human parathyroid hormone (1-34) [19], and denosumab [20]. In Europe, strontium ranelate [21] and recombinant human parathyroid hormone (1 - 84) [22] are available for treatment of osteoporosis; however, these agents are not currently FDAapproved for use in the USA. Potential future therapies with novel mechanisms of action that are currently in clinical trials include a cathepsin K inhibitor (odanacatib) [23] and two humanized mAbs to sclerostin (romosozumab, blosozumab) [24,25].

\section{Introduction to denosumab}

Denosumab is a human monoclonal IgG2 antibody genetically engineered in hamster ovary cells. It has high affinity and specificity for human receptor activator of nuclear factor kappa-B ligand (RANKL), the principle regulator of osteoclastic bone resorption. Denosumab binds to RANKL, preventing RANKL from activating its receptor, RANK, on the surface of osteoclasts and their precursors. This acts to inhibit osteoclast formation, function, and survival, thereby decreasing bone resorption.

\section{Pharmacodynamics}

In clinical studies reported by the manufacturer using subcutaneous (SC) denosumab $60 \mathrm{mg}$ dosing, C-telopeptide (CTX), a marker of bone resorption, was reduced by $85 \%$ at 3 days and below the limit of assay detection in 39\% of patients at 1 month in $68 \%$ of patients at 3 months. At the end of the 6 month dosing interval, CTX reductions were partially attenuated from maximal reduction of $\geq 87 \%-\geq 45 \%$ (range: $45-80 \%$ ), reflecting some reversibility of 
bone remodeling suppression. After discontinuation of denosumab therapy, markers of bone resorption increased to levels $40 \%-60 \%$ above pretreatment values, but returned to baseline levels within 12 months. Bone formation markers (osteocalcin and procollagen type $1 \mathrm{~N}$-terminal peptide [P1NP]) have been shown to be reduced by 1 month [26].

In a Phase I, dose-escalation clinical study of 49 healthy post-menopausal women given a single dose of denosumab [27] (0.01, 0.03, 0.1, 0.3, 1.0, and $3.0 \mathrm{mg} / \mathrm{kg}$ groups, $\mathrm{n}=6 /$ group $)$ versus placebo at a 3:1 ratio, decreases in urinary N-telopeptide (NTX, a marker of bone turnover) were observed as early as 12 hours post-dose with mean decrease of $77 \%$ in the $3.0 \mathrm{mg} / \mathrm{kg}$ group. Of note, the placebo group also showed a decrease of $46 \%$ at 12 hours, which was expected due to the diurnal variation of urinary NTX. However, at 24 hours after initial dosing, the mean decrease in urinary NTX was $73 \%$ in the $3.0 \mathrm{mg} / \mathrm{kg}$ groups versus only $10 \%$ in the placebo group. Maximum urinary NTX suppression was noted at 2 weeks for the $0.01,0.03,0.3$, and $1.0 \mathrm{mg} / \mathrm{kg}$ dose groups, 1 month for the $0.1 \mathrm{mg} / \mathrm{kg}$ group, and 3 months for the $3.0 \mathrm{mg} / \mathrm{kg}$ group (84\%). The treatment effect was reversible, with a return to baseline urinary NTX at 2 months for the 0.01 and $0.03 \mathrm{mg} / \mathrm{kg}$ dose groups, 4 months for the $0.1 \mathrm{mg} / \mathrm{kg}$ group, 6 months for the $0.3 \mathrm{mg} / \mathrm{kg}$ group, and 9 months for the 1.0 and $3.0 \mathrm{mg} / \mathrm{kg}$ groups, confirming a prolonged antiresorptive effect at higher doses.

In contrast to urinary NTX, bone-specific alkaline phosphatase did not rapidly decrease. A decrease from baseline occurred starting at 2 weeks, with maximum decrease of $53 \%$ at 5 months in the $3.0 \mathrm{mg} / \mathrm{kg}$ dose group.

The maximum decrease in albumin-adjusted serum calcium observed was 10\% (at 14 days in the $0.3 \mathrm{mg} / \mathrm{kg}$ group); no subjects had calcium levels below $2 \mathrm{mmol} / \mathrm{l}$. Serum phosphorus levels were also decreased, and serum intact parathyroid hormone (iPTH) levels showed an early, dose-dependent increase. Maximum increase in iPTH was $\sim$ threefold in the 3.0 $\mathrm{mg} / \mathrm{kg}$ dose group at 4 days post dose, with increases above baseline of $8 \%$ and $67 \%$ in the 1.0 and $3.0 \mathrm{mg} / \mathrm{kg}$ groups respectively at 6 months. Additionally, mean white blood cell count remained stable across all dose groups, with no changes in T and B cell counts (CD3, CD4, CD8, CD20, and CD56) or immunoglobulins. Binding antibodies were not detected.

\section{Pharmacokinetics and metabolism}

In numerous clinic trials, denosumab has demonstrated dose-dependent, non-linear pharmacokinetics across wide dose ranges, similar to other mAbs. It has a characteristic biochemical profile of prolonged absorption, prolonged $\beta$ phase, and a more rapid terminal phase [27]. In a study conducted in healthy male and female volunteers $(n=73$, age range: 18 to 64 years) following a single SC administered denosumab dose of $60 \mathrm{mg}$, the mean maximum denosumab concentration (Cmax) was $6.75 \mathrm{mcg} / \mathrm{ml}$ (standard deviation [SD] = $1.89 \mathrm{mcg} / \mathrm{ml}$ ), with a median time to maximum denosumab concentration (Tmax) was 10 days (range: 3 - 21 days), reflecting slow absorption by the SC route. After maximal concentration, serum denosumab concentrations declined over a period of $4-5$ months with a mean half-life of 25.4 days ( $\mathrm{SD}=8.5$ days; $\mathrm{n}=46$ ). No accumulation or change in denosumab pharmacokinetics with time was observed with serial dosing of $60 \mathrm{mg} \mathrm{SC}$ every 6 months [26]. 
A meta-analysis of 7 Phase I studies, 2 Phase II studies, and 2 Phase III studies $(n=1,564)$ determined the SC bioavailability of denosumab to be $64 \%$ with the first-order absorption rate constant $\mathrm{k}_{\mathrm{a}}=0.00883$ per hour, and the RANKL degradation rate was determined to be 0.00148 per hour. It was found that a $60 \mathrm{mg}$ fixed dose of denosumab given every 6 months provided similar RANKL inhibition as using weight-based dosing. Given that the nonlinear pharmacokinetic profile of denosumab is likely caused by RANKL binding, dosing adjustments based on age, gender, race, or body weight are not required [28].

In a study of 55 subjects with renal function ranging from normal to dialysis-dependent, it was concluded that denosumab does not require dose adjustment in patients with renal impairment, as no significant differences were seen in pharmacokinetic or pharmacodynamic parameters in this population [29].

Denosumab has not been evaluated in patients with hepatic impairment. Denosumab pharmacokinetics have not been shown to be affected by the formation of binding antibodies.

\section{Clinical efficacy (phase I, II, III studies)}

\subsection{Phase I study}

In a randomized, placebo-controlled, dose escalation study of denosumab in 49 healthy postmenopausal women, subjects were treated with a single SC dose of $0.01,0.03,0.1,0.3$, 1.0 , or $3.0 \mathrm{mg} / \mathrm{kg}$, or placebo, and monitored for drug safety, tolerability, pharmacokinetics, and effects on bone turnover markers (BTMs). Urinary NTX was suppressed, and a transient decrease in serum calcium ( $<10 \%$ on average) and a transient increase in serum iPTH (up to threefold after 4 days in the $3.0 \mathrm{mg} / \mathrm{kg}$ group) were noted. The treatment was well-tolerated with no drug-related serious adverse events reported [27].

\subsection{Phase II study}

In a 4-year Phase II randomized, placebo-controlled, dose-ranging study evaluating the efficacy and safety of denosumab in postmenopausal women with low bone mineral density (BMD), subjects were randomized to receive placebo, alendronate, or SC denosumab (q 3-6 months, dose ranges $6 \mathrm{mg}-210 \mathrm{mg}$ ) for the first 2 years, then re-allocated to continue, discontinue, or discontinue and reinitiate denosumab (60 mg q 6 month dosing); discontinue alendronate; or maintain placebo for 2 more years $(n=262)$ [30]. Denosumab was effective at increasing BMD (9.4-11.8\% increase at lumbar spine [LS] compared to $2.4 \%$ decrease at LS with placebo after 48 months) and suppressing BTMs in a rapid, sustained manner. Discontinuation of therapy led to return towards baseline values for both BMD (6.4\% decrease in BMD 12 months after discontinuation) and BTMs. BTM increases after discontinuation were generally a return to baseline values. BMD and BTM at baseline and 36 months (i.e., 24 months of denosumab and 12 months of discontinuation) were strongly correlated $(\mathrm{p}<0.0001)$. 


\subsection{Phase III studies}

FREEDOM (Fracture REduction Evaluation of Denosumab in Osteoporosis every 6 Months) was a three-year, Phase III clinical trial in 7868 postmenopausal women (age 6090 years) with osteoporosis ( $\mathrm{LS}$ or total hip T-score $<-2.5$ and not $<-4.0$ ) who were randomized to receive either SC denosumab $60 \mathrm{mg} \mathrm{q} 6$ months $(\mathrm{n}=3902)$ or placebo $(\mathrm{n}=$ 3906) (Table 1) [20]. The primary efficacy endpoint was new vertebral fractures at 36 months. The denosumab group had significant relative risk reduction for vertebral fractures (68\%), hip fractures (40\%), and non-vertebral fractures (20\%) compared with placebo. These data supported FDA approval of denosumab for the treatment of postmenopausal women with osteoporosis at high risk for fracture.

In the FREEDOM extension, which plans to follow patients for up to 10 years of denosumab therapy ( 7 years of extension beyond the initial FREEDOM trial), results currently are available up to 8 years of therapy (5 years of extension) (Table 1) [31]. At 8 years (1382 long-term, 1296 cross-over subjects from placebo group at year 3), BMD showed continued mean increases from baseline in the denosumab group: 18.5\% increase in LS and $8.2 \%$ increase in total hip in the long-term group, and $13.8 \%$ increase in the LS and $4.8 \%$ increase in the total hip in the cross-over group. New vertebral and non-vertebral fracture rate remained low throughout the extension, with a hip fracture rate during year 8 of $0.2 \%$ in long-term group and $0.1 \%$ in cross-over group.

The FREEDOM extension also addressed the sustainability of anti-fracture efficacy after therapy cessation by following patients who discontinued denosumab in an off-treatment observation period. This subgroup of 797 subjects (470 placebo, 327 denosumab) had completed 2-5 doses of denosumab versus placebo and was followed starting $>7$ months after their last dose, for up to 24 months of observation. During the off-treatment period (median 0.8 years per subject), $42 \%$ versus $28 \%$ of placebo- and denosumab-treated subjects, respectively, initiated other therapy. Following discontinuation, similar percentages of subjects in both groups sustained a new fracture (9\% placebo, $7 \%$ denosumab) [32].

A subset of the FREEDOM extension cohort included bone histology and histomorphometry evaluation. Transiliac crest bone biopsies in 41 subjected (13 cross-over, 28 long-term therapy) at year 2 of the extension showed normal bone quality, and a subset $(n=15)$ who were analyzed for dynamic parameters showed low bone turnover [33].

Several studies have compared the bone density response of denosumab to oral bisphosphonates, showing additional benefit with denosumab: DECIDE (Determining Efficacy: Comparison of Initiating Denosumab vs alendronate) [34] and STAND (Study of Transitioning from Alendronate to Denosumab) [35] trials of denosumab versus alendronate (Table 2), and trials of denosumab versus oral risedronate and ibandronate [36,37]. In the DECIDE trial, SC denosumab (60 mg q 6 months) plus weekly oral placebo versus weekly oral alendronate plus SC placebo injections q 6 months were compared over 12 months of use. At 12 months, denosumab showed a significantly greater BMD increase at the total hip compared to alendronate ( $3.5 \%$ denosumab, $2.6 \%$ alendronate, $\mathrm{p}<0.0001$ ), with treatment difference of $0.6 \%$ at femoral neck, and $1.1 \%$ at LS. Similar findings were reported with the STAND trial, which investigated denosumab versus alendronate in subjects already being 
treated with alendronate. At 12 months, there was a statistically significant greater increase in BMD with denosumab (1.9\% increase) compared with continuing alendronate $(1.05 \%$ increase), $p<0.0001$. In a comparison trial of denosumab versus risedronate, postmenopausal women who were previously treated with but suboptimally adherent to alendronate were randomized to receive denosumab SC q 6 months or oral risedronate 150 mg q month for 12 months. After 12 months of therapy, denosumab showed greater BMD gains at total hip ( $2.0 \%$ denosumab, $0.5 \%$ risedronate), femoral neck ( $1.4 \%$ denosumab, $0 \%$ risedronate), and lumbar spine ( $3.4 \%$ denosumab, $1.1 \%$ risedronate), $\mathrm{p}<0.0001$ at all sites. In a comparison trial of denosumab versus ibandronate, postmenopausal women who were previously treatment with bisphosphonates were randomized to receive denosumab SC q 6 months or oral ibandronate $150 \mathrm{mg} \mathrm{q}$ month for 12 months. The results of this trial were similar to the denosumab versus risedronate trial; after 12 months of therapy, denosumab showed greater BMD gains at total hip (2.3\% denosumab, $1.1 \%$ ibandronate), femoral neck ( $1.7 \%$ denosumab, $0.7 \%$ ibandronate), and lumbar spine (4.1\% denosumab, $2.0 \%$ ibandronate); $\mathrm{p}<0.0001$ at all sites.

ADAMO (Study to Compare the Efficacy and Safety of DenosumAb Versus Placebo in Males with Osteoporosis) was a randomized controlled trial (RCT) upon which the FDA based its approval for the use of denosumab in the treatment of men with osteoporosis at high risk for fracture. In this study, 242 men ages 30-85 with osteoporosis were randomized to receive denosumab vs. placebo ( $\mathrm{n}=121$ in each group) (Table 1) [38]. The primary endpoint of the study was to assess percentage change from baseline in LS BMD at 12 months. After 12 months, denosumab resulted in BMD increases of 5.7\% at the LS, 2.4\% at the total hip, and $2.1 \%$ at the femoral neck (adjusted $\mathrm{p} \leq 0.0144$ for BMD percent differences at all sites compared with placebo). These results remained robust on further analyses controlling for baseline covariates, such as baseline testosterone levels, BMD Tscores, and 10-year osteoporotic fracture risk.

The Hormone Ablation Bone Loss Trial (HALT) was an RCT of denosumab use in men undergoing androgen-deprivation therapy for non-metastatic, hormone-sensitive prostate cancer resulting in FDA approval for use for that indication (Table 1) [39]. Subjects with prostate cancer on androgen deprivation therapy were randomized to denosumab versus placebo for 24 months ( $\mathrm{n}=734$ in each group). The primary end point was the percent change in the baseline BMD of the LS at 24 months. At 24 months, BMD of the LS had increased by $5.6 \%$ in the denosumab group as compared with a loss of $1.0 \%$ in the placebo group $(\mathrm{p}<$ 0.001 ); significant differences between the two groups were seen at as early as 1 month and sustained through 36 months. Subjects who received denosumab had a decreased incidence of new vertebral fractures at 36 months $(1.5 \%$, vs. $3.9 \%$ with placebo, relative risk, 0.38 ; $95 \%$ CI, $0.19-0.78 ; \mathrm{p}=0.006$ ).

A 2-year RCT of denosumab was conducted in women with hormone receptor-positive nonmetastatic breast cancer treated with adjuvant aromatase inhibitor therapy; this trial led to FDA approval for use to increase bone mass in women at high risk for fracture receiving adjuvant aromatase inhibitor therapy for breast cancer (Table 1) [40]. Subjects were randomized to SC denosumab $60 \mathrm{mg}$ q 6 months $(\mathrm{n}=127)$ versus placebo $(\mathrm{n}=125)$, with the primary end point of percentage change in LS BMD from baseline to month 12. LS 
BMD increased by $5.5 \%$ at 12 months and $7.6 \%$ at 24 months greater in the denosumab group versus the placebo group ( $\mathrm{p}<0.0001$ at both time points). BMD increases were not influenced by duration of aromatase inhibitor therapy.

DATA (the Denosumab and Teriparatide Administration study) was a 2-year RCT in 94 postmenopausal women with osteoporosis in which subjects were randomized in a 1:1:1 ratio to receive $20 \mathrm{mcg}$ teriparatide daily, $60 \mathrm{mg}$ SC denosumab q 6 months, or both (Table 1) [41]. At 12 months, LS BMD increased more in the combination group (9.1\%, [SD 3.9]) than in the teriparatide $(6.2 \%$ [4.6], $\mathrm{p}=0.0139)$ or denosumab $(5.5 \%$ [3.3], $\mathrm{p}=0.0005)$ groups, as did total-hip BMD (combination, 4.9\% [2.9]; teriparatide, 0.7\% [2.7], p < 0.0001; denosumab 2.5\% [2.6], $\mathrm{p}=0.0011$ ). CTX showed maximal suppression in the denosumab and combination groups, and bone formation markers (osteocalcin, P1NP) remained measurable in the combination group in contrast to un-measureable in the denosumab group. These findings are in contrast to studies combining bisphosphonates with teriparatide, which have shown poorer efficacy with combined therapy compared to monotherapy $[42,43]$. These studies show less suppression of BTMs with combination than with bisphosphonate monotherapy, unlike the DATA trial, which showed maximum suppression of BTMs in both denosumab monotherapy and combination therapy. This effect difference may be due to more effective un-linking of bone resorption and formation with denosumab compared to bisphosphonates; denosumab appears to suppress teriparatide-induced bone resorption while only partially reducing teriparatide-induced bone formation.

The DAPS (Denosumab Adherence Preference Satisfactions) study was a 2-year RCT, which analyzed patient adherence and satisfaction in 221 postmenopausal women with osteoporosis, treated with either denosumab or alendronate in alternating years (Table) [44]. Less non-adherence to therapy was found in the denosumab group versus alendronate (first year, $11.9 \%$ vs. $23.4 \%$; second year, $7.5 \%$ vs. $36.5 \%$ ), and $92.4 \%$ of subjects expressed preference for denosumab over alendronate.

There are numerous ongoing Phase III trials investigating denosumab use in osteoporosis, including denosumab use for prevention of post-teriparatide bone loss (NCT02166437), denosumab use in glucocorticoid-induced osteoporosis (NCT01465568), denosumab compared with zoledronic acid in women with postmenopausal osteoporosis (NCT01732770), and investigations for use in thalassemia-induced osteoporosis [45] (clinicaltrials.gov).

\section{Safety, tolerability, and clinical monitoring}

Due to a theoretical risk of impaired immune response and profound decreases of BTMs with RANKL inhibition, the long-term safety profile of denosumab has been carefully scrutinized in ongoing extension trials. With an estimated exposure to denosumab of 1,252,566 patient-years as of September 2013, there have been post-marketing reports of atypical femur fractures (AFF), osteonecrosis of the jaw (ONJ), severe symptomatic hypocalcemia (SSH), and anaphylaxis. Four cases of AFF consistent with American Society for Bone and Mineral Research definitions have been adjudicated; all patients had prior bisphosphonate exposure and suffered AFF within 18 months of exposure to denosumab. 
Thirty-two cases of ONJ consistent with American Association of Oral and Maxillofacial Surgeons definitions have been adjudicated. Risk factors for ONJ in this population included use of glucocorticoids/chemotherapy, prior bisphosphonate use, older age, and invasive dental procedures.

Eight cases of SSH were reported including symptoms of seizure and/or tetany; seven of eight of these patients had chronic kidney disease, a known risk factor for hypocalcemia. Anaphylaxis was reported in five patients; there were no fatal outcomes from this complication [46]. Although anaphylaxis and allergic reactions are rare, it would be useful for the manufacturer to provide a test dose of this denosumab.

Significant differences between denosumab treatment and placebo were seen with skinrelated conditions in the parent FREEDOM trial (3\% denosumab vs $1.7 \%$ placebo), with cellulitis as a serious adverse event (AE) more common with denosumab (12 subjects denosumab vs one subject placebo). In the DEFEND (DEnosumab FortifiEs boNe Density) trial $(n=330)$, the overall incidence of infectious AEs were similar (60\% denosumab, $61 \%$ placebo); however more serious infections were reported in the denosumab group (eight denosumab, one placebo). These included pneumonia, diverticulitis, appendicitis, sepsis, pyelonephritis, urinary tract infection, and cellulitis in the denosumab group, versus lobar pneumonia in the placebo group [47]. However, in the FREEDOM extension, rates of all, serious, and fatal AEs in the long-term denosumab group were similar to or lower than the parent trial rates. The serious skin infection rate with denosumab was similar to the placebo group $(<0.1 / 100$ subject years in both groups), and the infection rate was lower with denosumab during the extension compared with placebo in the FREEDOM parent study (23.4/100 subject years vs 30.7/100 subject years respectively) [48]. However, an earlier meta-analysis of three Phase II and III studies [49] showed a relative risk of infectious serious AE of 4.45 (95\% CI $1.15-17.14, \mathrm{p}=0.03)$ at 24 months of therapy $(\mathrm{n}=17$ infectious serious $\mathrm{AE}$ in combined denosumab arms, $\mathrm{n}=$ two infectious serious $\mathrm{AE}$ in combined placebo arms). When combined with data from the parent FREEDOM trial, the relative risk of infectious serious AEs decreased to 2.10 (95\% CI $0.64-6.9, \mathrm{p}=0.22$ ), which was not statistically significant compared to placebo [50]. This meta-analysis also reported no statistically significant increased risk of neoplasm (relative risk 1.11, 95\% CI $0.91-1.36, \mathrm{p}=0.30$ ), which was an initial concern when denosumab was first marketed. Studies in prevention of skeletal-related events in multiple myeloma have yielded less favorable results, though data is limited [51]. In a Phase III trial comparing denosumab with zoledronic acid in multiple myeloma patients with at least 1 osteolytic lesion, denosumab did not reach superiority (though non-inferiority was achieved), and a post-hoc analysis showed less favorable survival in the denosumab group (HR 2.26, CI $1.13-4.50$ ), though AEs were similar between groups [52]. Due to this finding, denosumab is not currently approved for use in multiple myeloma.

The manufacturer of denosumab recommends all patients using denosumab to receive calcium $1000 \mathrm{mg}$ daily and at least $400 \mathrm{IU}$ of vitamin D daily to avoid hypocalcemia complications. Monitoring of calcium, magnesium, and phosphorus in patients with chronic kidney disease is highly recommended. Denosumab is currently contraindicated for use in 
those with concurrent hypocalcemia, during pregnancy (category X), and in those with history of hypersensitivity reaction to denosumab [26].

\section{Regulatory affairs}

The initial U.S. FDA approval for denosumab was in June 2010 for the indication of treatment of osteoporosis in post-menopausal women at high risk for fracture. In September 2011, the FDA granted an additional indication for treatment to increase bone mass in men at high risk for fracture receiving androgen deprivation therapy for non-metastatic prostate cancer, and to increase bone mass in women at high risk for fracture receiving adjuvant aromatase inhibitor therapy for breast cancer. In September 2012, denosumab was approved to increase bone mass in men with osteoporosis at high risk for fracture. The FDA has defined high risk for fracture for both men and women with postmenopausal osteoporosis as a history of osteoporotic fractures, or multiple risk factors for fracture, or patients who have failed or are intolerant to other available osteoporosis therapy.

In addition to FDA approval in the USA, denosumab is currently approved for use in the treatment of osteoporosis in all 27 European Union countries, Canada, and Australia.

\section{Conclusion}

Denosumab is the first and so far the only RANKL inhibitor approved for treatment of osteoporosis. It is extremely effective at decreasing bone turnover and has reversible effects on discontinuation. Since its approval, it has shown sustained efficacy in increasing BMD and decreasing fracture risk, with extension data now through 8 years of use. Though safety has been a concern for this agent given its novel mechanism, current post-marketing data appear favorable in this aspect, showing good tolerability and safety compared to placebo and bisphosphonates.

\section{Expert Opinion}

Denosumab is becoming an increasingly utilized therapy in treating osteoporosis and bone density loss in the USA; much of this is due to continued favorable data showing significant efficacy, overall low adverse events, and convenient dosing. The FREEDOM extension has shown no new safety concerns arising in its use up to 8 years. A major benefit of denosumab over the standard therapy with bisphosphonates is a continuous BMD increase with use as opposed to a plateau effect seen with bisphosphonates, and the possibility of combination with teriparatide for further BMD gains.

Although denosumab has some safety concerns, such as AFF and ONJ, in common with bisphosphonates, reassuring characteristics of denosumab include its lack of accumulation in bone and reversibility of antiresorptive effects shortly following the end of the 6 month dosing interval $[26,30]$. This rapid "offset" of antiresorptive effect in 6 months contrasts with prolonged persistence of anti-resorption following discontinuation of long-term therapy with commonly used bisphosphonates, particularly alendronate and zoledronic acid. Given the many years of therapy that may be necessary for patients at high risk for fracture, denosumab may prove safer for prolonged periods of use. However, this theoretical safety 
benefit has yet to be proven; furthermore, the rapid reversibility of antiresorptive effect with discontinuation of denosumab raises concern of adverse clinical consequences and increased fracture risk in patients who do not return for treatment at regular 6-month intervals. Cessation of denosumab results in an increase of BTMs back to, and transiently above, baseline levels. It is not known whether this time period of accelerated bone turnover is associated with an increase in fracture risk. Non-compliant patients would be at increased risk of this phenomenon, leading to increased need for patient monitoring for adherence. Further, questions remain on how to transition patients off of denosumab, without resulting in an increased in BTMs. However, studies have shown overall good adherence to and patient satisfaction with denosumab compared to oral bisphosphonates, which may mitigate this concern [44].

Another major safety concern is infection risk, given RANKL inhibition of non-skeletal immune cells, causing a theoretical immune suppression. The extent of this risk remains unclear; although in FREEDOM extension the infection rates were similar to placebo [48], the initial FREEDOM data showed higher frequency of endocarditis (three in denosumab vs. zero in placebo group), and severe skin events $(0.4 \%$ in denosumab vs. $<0.1 \%$ in placebo group, $\mathrm{p}<0.05$ ), suggesting a potential, but not definitive, increased risk of infections in immunocompromised patients [53]. Of note, rates of opportunistic infections were similar between denosumab and placebo groups.

\section{Bibliography}

Papers of special note have been highlighted as either of interest $(\bullet)$ or of considerable interest $(\bullet)$ to readers.

1. Wright N, Looker A, Saag K, et al. The recent prevalence of osteoporosis and low bone mass in the United States based on bone mineral density at the femoral neck or lumbar spine. J Bone Miner Res. 2014; 29(10):2520-6. [PubMed: 24771492]

2. Burge R, Dawson-Hughes B, Solomon D, et al. Incidence and economic burden of osteoporosisrelated fractures in the United States, 2005-2025. J Bone Miner Res. 2007; 22(3):465-75. [PubMed: 17144789]

3. Hannan E, Magaziner J, Wang J, et al. Mortality and locomotion 6 months after hospitalization for hip fracture: risk factors and risk-adjusted hospital outcomes. JAMA. 2001; 285:2736-42. [PubMed: 11386929]

4. Cooper C, Atkinson EJ, Jacobsen SJ. Population-based study of survival after osteoporotic fractures. Am J Epidemiol. 1993; 137:1001-5. [PubMed: 8317445]

5. Roux C, Wyman A, Hooven F, et al. Burden of non-hip, non-vertebral fractures on quality of life in postmenopausal women. Osteoporos Int. 2012; 23(12):2863-71. [PubMed: 22398855]

6. Dawson-Hughes B, Looker A, Tosteson A, et al. The potential impact of new National Osteoporosis Foundation guidance on treatment patterns. Osteoporos Int. 2010; 21(1):41-52. [PubMed: 19705046]

7. US Department of Health and Human Services. Bone Health and Osteoporosis: A Report of the Surgeon General. US Department of Health and Human Services, Office of the Surgeon General; 2004.

8. Kothawala P, Badamgarav E, Ryu S, et al. Systematic review and meta-analysis of real-world adherence to drug therapy for osteoporosis. Mayo Clin Proc. 2007; 82(12):1493-501. [PubMed: 18053457]

9. Food and Drug Administration. Background document for meeting of Advisory Committee for Reproductive Health Drugs and Drug Safety and Risk Management Advisory Committee. 2011 
10. Liberman U, Weiss S, Broll J, et al. Effects of oral alendronate on bone mineral denisty and the incidence of fracture in postmenopausal osteoporosis. N Engl J Med. 1995; 333(22):1437-43. [PubMed: 7477143]

11. Mcclung M, Geusens P, Miller P, et al. Effects of risedronate on the risk of hip fracture in elderly women. Hip Intervention Program Study Group N Engl J Med. 2001; 344(5):333-40.

12. Chestnut CI, Skag A, Christiansen C, et al. Effects of oral ibandronate administered daily or intermittently on fracture risk in postmenopausal osteoporosis. J Bone Miner Res. 2004; 19(8): 1241-9. [PubMed: 15231010]

13. Black D, Delmas P, Eastell R, et al. Once-yearly zoledronic acid for treatment of postmenopausal osteoporosis. N Engl J Med. 2007; 356(18):1809-22. [PubMed: 17476007]

14. Chestnut CI, Silverman S, Andriano K, et al. A randomized trial of nasal spray salmon calcitonin in postmenopausal women with established osteoporosis: the prevent recurrence of osteoporotic fractures study. Am J Med. 2000; 109(4):267-76. [PubMed: 10996576]

15. Rossouw J, Anderson G, Prentice R, et al. Risks and benefits of estrogen plus protestin in healthy postmenopausal women: principal results From the Women's Health Initiative randomized controlled trial. JAMA. 2002; 288(3):321-33. [PubMed: 12117397]

16. Anderson G, Limacher M, Assaf A, et al. Effects of conjugated equine estrogen in postmenopausal women with hysterectomy: the Women's Health Initiative randomized controlled trial. JAMA. 2004; 291(14):1701-12. [PubMed: 15082697]

17. Ettinger B, Black D, Mitlak B, et al. Reduction of vertebral fracture risk in postmenopausal women with osteoporosis treated with raloxifene: results from a 3-year randomized clinical trial. Multiple Outcomes of Raloxifene Evaluation (MORE) Investigators JAMA. 1999; 282(7):637-45.

18. Lindsay R, Gallagher J, Kagan R, et al. Efficacy of tissue-selective estrogen complex of bazedoxifene/conjugated estrogens for osteoporosis prevention in at-risk postmenopausal women. Fertil Steril. 2009; 92(3):1045-52. [PubMed: 19635616]

19. Neer R, Arnaud C, Zanchetta J, et al. Effect of parathyroid hormone (1-34) on fractures and bone mineral density in postmenopausal women with osteoporosis. N Engl J Med. 2001; 344(19):143441. [PubMed: 11346808]

20••. Cummings S, San Martin J, Mcclung M, et al. Denosumab for prevention of fractures in postmenpausal women with osteoporosis. N Engl J Med. 2009; 361(8):756-65. The FREEDOM trial, led to approval for denosumab use in treatment of post-menopausal women at high risk for fracture. Showed denosumab reduced risk of vertebral, hip, and nonvertebral fractures in 7868 postmenopausal women with osteoporosis. [PubMed: 19671655]

21. O’Donnell S, Cranney A, Wells G, et al. Strontium ranelate for preventing and treating postmenopausal osteoporosis. Cochrane Database Syst Rev. 2006; 19(3):CD005326.

22. Greenspan S, Bone H, Ettinger M, et al. Effect of recombinant human parathyroid hormone (1-84) on vertebral fracture and bone mineral density in postmenopausal women with osteoporosis: a randomized trial. Ann Intern Med. 2007; 146(5):326. [PubMed: 17339618]

23. Bone H, McClung M, Roux C, et al. Odanacatib, a cathepsin-K inhibitor for osteoporosis: a twoyear study in postmenopausal women with low bone density. J Bone Miner Res. 2010; 25(5):93747. [PubMed: 19874198]

24. Padhi D, Jang G, Stouch B, et al. Singledose, placebo-controlled, randomized study of AMG 785, a sclerostin monoclonal antibody. J Bone Miner Res. 2011; 26(1):19-26. [PubMed: 20593411]

25. Recker R, Benson C, Matsumoto T, et al. A randomized, double-blind phase 2 clinical trial of blosozumab, a sclerostin antibody, in postmenopausal women with low bone mineral density. J Bone Miner Res. 2014 [Epub ahead of print].

26. Amgen, Inc. Prolia (denosumab) prescribing information. 2010 updated 2014.

27. Bekker P, Holloway D, Rasmussen A, et al. A single-dose placebo-controlled study of AMG 162, a fully monoclonal antibody to RANKL, in postmenopausal women. J Bone Miner Res. 2004; 19(7): 1059-66. [PubMed: 15176987]

28. Sutjandra L, Rodriguez R, Doshi S, et al. Population pharmacokinetic metaanalysis of denosumab in healthy subjects and postmenopausal women with osteopenia or osteoporosis. Clin Pharmacokinet. 2011; 50(12):793-807. [PubMed: 22087866] 
29. Block G, Bone H, Fang L, et al. A single-dose study of denosumab in patients with various degrees of renal impairment. J Bone Miner Res. 2012; 27(7):1471-9. [PubMed: 22461041]

30. Miller P, Bolognese M, Lewiecki E, et al. Effect of denosumab on bone density and turnover in postmenopausal women with low bone mass after long-term continued, discontinued, and restarting of therapy: a randomized blinded phase 2 clinical trial. Bone. 2008; 43(2):222-9. [PubMed: 18539106]

31••. Papapoulos, S.; Lippuner, K.; Roux, C., et al. ASBMR 2013 Annual Meeting. 2013. Eight years of denosumab treatment in postmenopausal women with osteoporosis: results from the first five years of the FREEDOM extension. Oral Poster Session; Presentation Number: LB-MO26Results from the FREEDOM extension, up to 8 years of continuous therapy with denosumab, which showed BMD gains of $18.5 \%$ in LS and $8.2 \%$ in total hip in the longterm group.

32. Brown J, Roux C, Torring O, et al. Discontinuation of denosumab and associated fracture incidence: analysis from the Fracture Reduction Evaluation of Denosumab in Osteoporosis Every 6 Months (FREEDOM) trial. J Bone Miner Res. 2013; 28(4):746-52. [PubMed: 23109251]

33. Brown J, Reid I, Wagman R, et al. Effects of up to 5 Years of Denosumab Treatment on Bone Histology and Histomorphometry: the FREEDOM Study Extension. J Bone Miner Res. 2014; 29(9):2051-6. [PubMed: 24692050]

34. Brown J, Prince R, Deal C, et al. Comparison of the effect of denosumab and alendronate on BMD and biochemical markers of bone turnover in postmenopausal women with low bone mass: a randomized, blinded, phase 3 trial. J Bone Miner Res. 2009; 24(1):153-61. [PubMed: 18767928]

35. Kendler D, Roux C, Benhamou C, et al. Effects of denosumab on bone mineral density and bone turnover in postmenopausal women transitioning from alendronate therapy. J Bone Miner Res. 2009; 25(1):72-81. [PubMed: 19594293]

36. Roux C, Hofbauer L, Ho P, et al. Denosumab compared with risedronate in postmenopausal women suboptimally adherent to alendronate therapy: efficacy and safety results from a randomized open-label study. Bone. 2014; 58:48-54. [PubMed: 24141036]

37. Recknor C, Czerwinski E, Bone H, et al. Denosumab compared with ibandronate in postmenopausal women previously treated with bisphosphonate therapy: a randomized open-label trial. Obstet Gynecol. 2013; 121(6):1291-9. [PubMed: 23812464]

38. Orwoll E, Teglbjærg C, Langdahl B, et al. A randomized, placebo-controlled study of the effects of denosumab for the treatment of men with low bone mineral density. J Clin Endocrinol Metab. 2012; 97(9):3161-9. [PubMed: 22723310]

39•. Smith M, Egerdie B, Toriz N, et al. Denosumab in men receiving androgendeprivation therapy for prostate cancer. N Engl J Med. 2009; 361(8):745-55. Study of denosumab use for bone loss in men with nonmetastatic prostate cancer receving androgen-deprivation therapy, which led to approval for use in this population. [PubMed: 19671656]

40•. Ellis GK, Bone HG, Chlebowski R, et al. Randomized trial of denosumab in patients receiving adjuvant aromatase inhibitors for nonmetastatic breast cancer. J Clin Oncol. 2008; 26:4875-82. Study of denosumab use for bone loss in women with breast cancer receiving adjuvant aromatase inhibitor therapy, which led to approval of denosumab use in this population. [PubMed: 18725648]

41. Tsai J, Uihlein A, Lee H, et al. Teriparatide and denosumab, alone or combined, in women with postmenopausal osteoporosis: the DATA study randomised trial. Lancet. 2013; 382(9886):50-6. [PubMed: 23683600]

42. Cosman F, Eriksen E, Recknor C, et al. Effects of intravenous zoledronic acid plus subcutaneous teriparatide [rhPTH (1-34)] in postmenopausal osteoporosis. J Bone Miner Res. 2011; 26(3):50311. [PubMed: 20814967]

43. Finkelstein J, Wyland J, Lee H, et al. Effects of teriparatide, alendronate, or both in women with postmenopausal osteoporosis. J Clin Endocrinol Metab. 2010; 95(4):1838-45. [PubMed: 20164296]

44. Freemantle N, Satram-Hoang A, Tang E, et al. Final results of the DAPS (Denosumab Adherence Preference Satisfaction) study: a 24-month, randomized, crossover comparison with alendronate in postmenopausal women. Osteoporos Int. 2012; 23(1):317-26. [PubMed: 21927922] 
45. Yassin M, Soliman A, De Sanctis V, et al. Effects of the anti-receptor activator of nuclear factor kappa B ligand denusomab on beta thalassemia majorinduced osteoporosis. Indian J Endocrinol Metab. 2014; 18(4):546-51. [PubMed: 25143915]

46. Geller M, Wagman R, Ho P, et al. Early findings from Prolia_post-marketing safety surveillance for atypical femur fracture, osteonecrosis of the jaw, severe symptomatic hypocalcemia, and anaphylaxis. Ann Rheum Dis. 2014; 73:Suppl2.

47. Bone H, Bolognese M, Yuen C, et al. Effects of denosumab on bone mineral density and bone turnover in postmenopausal women. J Clin Endocrinol Metab. 2008; 93(6):2149-57. [PubMed: 18381571]

48. Bone H, Chapurlat R, Brandi M, et al. The effect of three or six years of denosumab exposure in women with postmenopausal osteoporosis: results from the FREEDOM extension. J Clin Endocrinol Metab. 2013; (11):4483-92. [PubMed: 23979955]

49. Anastasilakis A, Toulis K, Goulis D, et al. Efficacy and safety of denosumab in postmenopausal women with osteopenia or osteoporosis: a systematic review and a meta-analysis. Horm Metab Res. 2009; 41(10):721-9. [PubMed: 19536731]

50. von Keyserlingk C, Hopkins R, Anastasilakis A, et al. Clinical efficacy and safety of denosumab in postmenopausal women with low bone mineral density and osteoporosis: a meta-analysis. Semin Arthritis Rheum. 2011; 41(2):178-86. [PubMed: 21616520]

51. Hageman K, Patel K, Mace K, et al. The role of denosumab for prevention of skeletal-related complications in multiple myeloma. Ann Pharmacother. 2013; 47(7-8):1069-74. [PubMed: 23780806]

52. Henry D, Costa L, Goldwasser F, et al. Randomized, double-blind study of denosumab versus zoledronic acid in the treatment of bone metastases in patients with advanced cancer (excluding breast and prostate cancer) or multiple myeloma. J Clin Oncol. 2011; 29(9):1125-32. [PubMed: 21343556]

53. Watts N, Roux C, Modlin J, et al. Infections in postmenopausal women with osteoporosis treated with denosumab or placebo: coincidence or causal association? Osteoporos Int. 2012; 23(1):32737. [PubMed: 21892677]

54. Black D, Cummings S, Karpf D, et al. Randomised trial of effect of alendronate on risk of fracture in women with existing vertebral fractures. Fracture Intervention Trial Research Group Lancet. 1996; 348(9041):1535-41.

55. Black D, Delmas P, Eastell R, et al. Once-yearly zoledronic acid for treatment of postmenopausal osteoporosis. N Engl J Med. 2007; 356(18):1809-22. [PubMed: 17476007] 
Box 1

\section{Drug summary}

Drug Name

Phase

Approved Indications

Route of

administrationChemical

structure

Pivotal trials
Denosumab

Post-marketing

Treatment of postmenopausal women with osteoporosis at high risk for fracture;

Treatment to increase bone mass in men with osteoporosis at high risk of fracture;

Treatment to increase bone mass in men at high risk for fracture receiving androgen deprivation therapy for non-metastatic prostate cancer;

Treatment to increase bone mass in women at high risk for fracture receiving adjuvant aromatase inhibitor therapy for breast cancer

Suppression of bone resorption by binding to RANK ligand, preventing its binding to RANK and decreasing osteoclast formation, activity, and survival

Subcutaneous every 6 months(C6404 H9912 N1724 O2004 S50) consists of 2 heavy and 2 light chains; each light chain consists of 215 amino acids and each heavy chain consists of 448 amino acids with 4 intramolecular disulfides. Fully human monoclonal IgG2 antibody to RANK ligand

FREEDOM, ADAMO 


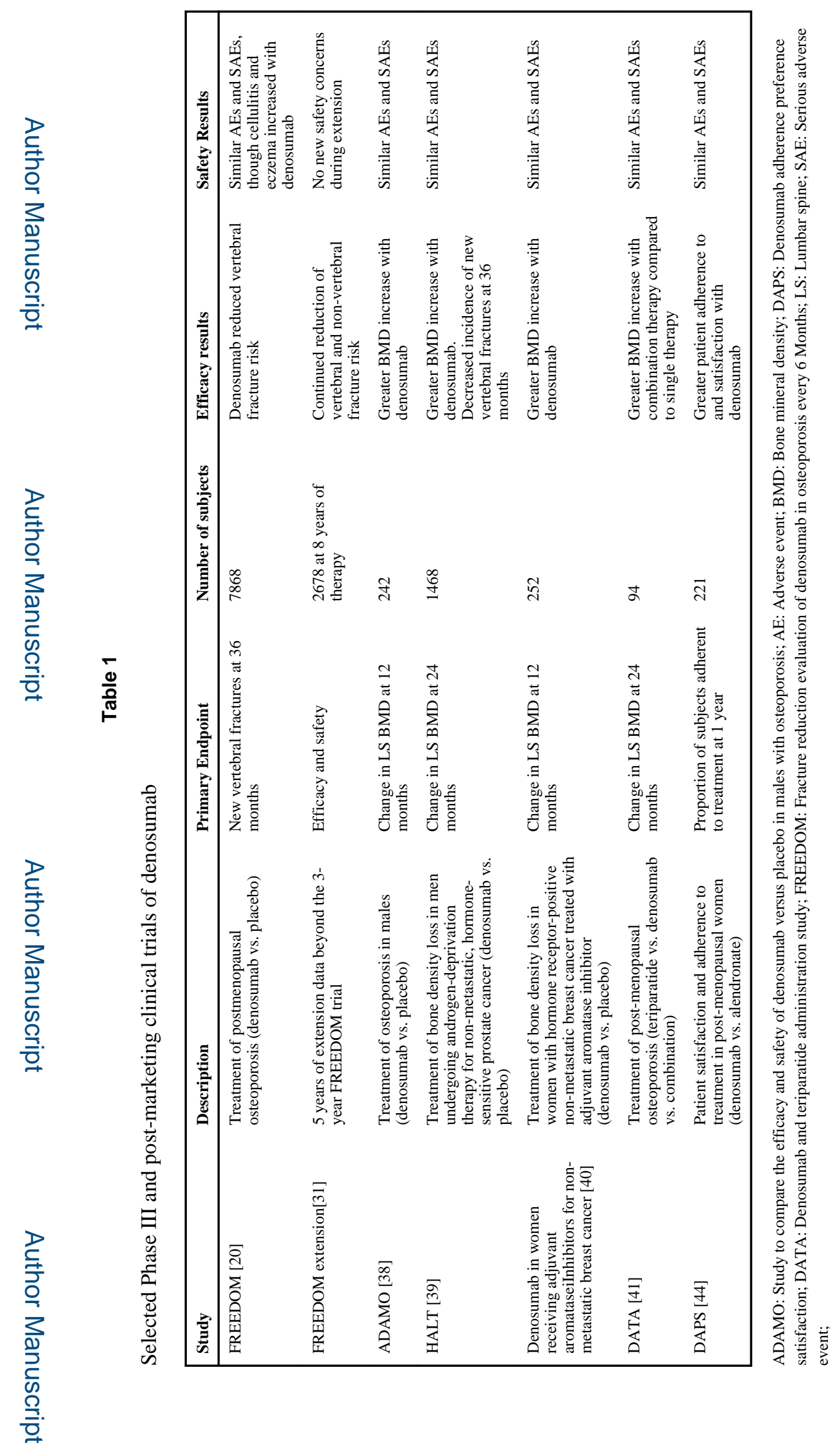

Expert Opin Drug Metab Toxicol. Author manuscript; available in PMC 2016 March 01. 
Table 2

Comparison of major characteristics of bisphosphonates (alendronate and zoledronic acid) versus denosumab.

Bisphosphonates

Alendronate

Efficacy

Pivotal Trial

Safety

Administration

Pharmacokinetics

Pharmacodynamics

Use in renal

impairment

Contraindicated for GFR $<35$

Poor oral bioavailability. Nonuniform drug accumulation within bone matrix, with elimination halflife of $\sim 10$ years

Slow suppression of bone turnover

Vertebral and hip fracture reduction: 50\% (STAND an

DECIDE trials comparing greater BMD gains with

denosumab at 12 months)

FIT [54]

Risk for AFF and ONJ with prolonged exposure

Oral, patient administered weekly

Relative cost
Low (generic available)

Zoledronic Acid

Vertebral fracture reduction: $70 \%$. Hip fracture reduction: $41 \%$. (Ongoing trial comparing efficacy of ZA vs denosumab)

\section{HORIZON-PFT [55]}

Risk for $\mathrm{AFF}$ and $\mathrm{ONJ}$ with exposure to high doses and/or prolonged exposure

Intravenous administration once a year. Requires IV catheter placement

Non-uniform drug accumulation within bone matrix, with prolonged suppression of bone turnover

More rapid suppression of bone turnover than oral (within days)

Contraindicated for GFR $<35$

Moderate/High (generic available) $68 \%$. Hip fracture reduction: $40 \%$

Denosumab

\section{FREEDOM [20]}

Risk for AFF and ONJ.

Increased risk of serious skin

infections

Subcutaneous injection every 6 months. Can be administered in office

No bone accumulation, effects reversible by $9-12$ months post-dose

Rapid suppression of bone turnover (within $12 \mathrm{~h}$ )

No restriction for use in renal impairment, though close monitoring of serum calcium in patients with GFR $<30$ recommended

High

AFF: Atypical femur fractures; GFR: Glomerular filtration rate; ONJ: Osteonecrosis of the jaw. 